\title{
Research on Selection of Shape Function in Flexible Multi-body System Dynamics
}

\author{
Yang Zhao ${ }^{1,}$, , Qizheng Zhou ${ }^{2, b}$ and Pengfei Yue ${ }^{3, c}$ \\ Department of Weaponry Engineering,Naval University of Engineering,Hubei Wuhan 430033,China \\ a1573380179@qq.com, bzqizheng@126.com, cypenfei@163.com
}

\begin{abstract}
Keywords: Flexible multi-body system dynamics;Shape function;Mode coordinates;Static deformation;Floating coordinate system

Abstract:In order to improve the accuracy and efficiency of flexible multibody system dynamics simulation and control, the research on the selection of shape function in flexible multi- body system is needed.Under the specific floating coordinate system, deduce flexible multi-body system dynamics general equation based on Lagrange equation. Analysis and compare the coefficient matrix of dynamic equation In view of the three different types of shape function, the effect of shape function selection for dynamics calculation accuracy and efficiency is studied.Research shows that floating coordinate system affect the degree of equation coupling, and The main factors influencing the calculation accuracy and efficiency is shape functionThe form of Dynamics equation under static deformation assumption is the simplest and calculation efficiency is highest.Calculation efficiency of the finite element model and vibration mode model increases as the number of units and the number of mode truncation decreases.Calculation efficiency and accuracy interact on each other and determine the shape function of the form.
\end{abstract}

\section{Introduction}

The flexible multi-body system dynamics focuses on the research on component elasticity in mechanical and structural systems, motion pair deformation and coupling dynamic characteristics of rigid motion, which has the same research purpose with the multi-rigid-body dynamics, namely, it aims to solve two problems of simulation and forward \& inverse control of the dynamic system ${ }^{[1]}$. In the process of developing rigid system dynamics, the algorithm efficiency needed for real-time control and simulation calculation should be first concerned, thus various recursive algorithms ${ }^{[2-4]}$ are established by analyzing multiple principles of mechanics ${ }^{[2-5]}$. With the progress in computer and chip technology, the efficiency of the algorithm is no longer the research focus, and the closed kinetic equation of the system in general form can be obtained by no matter what kind of principle according to the equivalence of mechanical principles ${ }^{[1,5]}$. In various fields such as robot ${ }^{[6]}$, spacecraft ${ }^{[7]}$, sport biomechanics $^{[8]}$ and gun mechanism ${ }^{[9-13]}$, the needed dynamic model can be established with the theory and algorithm of rigid multi-body dynamics, which is used for the analysis of the system response or the design of dynamic control algorithm for the system.

The further consideration of the deformation characteristic of member or hinge in the system based on rigid multi-body dynamics is the demand in the engineering, and the theory for the improvement of the accuracy of simulation and control algorithm. Zhou Qizhao indicates in the Article $^{[13]}$ that the system is stable if the panel of the spacecraft with the solar panel is considered as the rigid body, while the system may be instable when the elasticity of the panel is considered. In addition, at the early stage of the development of flexible body system dynamics, the important research problems of flexible multi-body system dynamics are pointed out ${ }^{[14]}$. The first problem is the selection of the floating coordinate system, which decides the coupling degree of the kinetic equation of the system ${ }^{[1,13,14,15]}$; The second is the description of the deformation of elastomer or hinge, which decides the computational efficiency and computational accuracy of the simulation and control algorithm ${ }^{[1,14]}$. These problems caused by shape function of floating coordinate system and the elastomer seem relatively perfect in theory; however, the problem in the engineering field is that how to select them aiming at simulation and control problems in certain field is not clear enough. In terms of simulation and control in various fields such as spacecraft and gun at present, the dynamics model 
is generally established in a given way, and the correlation algorithm is designed after the simplified treatment. In fact, aiming at different application situations, the requirements for the establishment of floating coordinates as well as the deformation of the elastomer and its control are different, especially the selection of the shape function, and the requirements for the accuracy in different application fields decide the complexity of the assumed shape function and the computational efficiency. For example, it is unnecessary to select the shape function of the same computational accuracy for the deformation of the gun barrel and the solar panel of the spacecraft. These problems are not studied thoroughly now.

In this paper, aiming at the problem that the shape function affects computational accuracy and efficiency of figures, and based on the analysis of the floating coordinate system established at the specified hinge, the kinetic equation of the flexible multi-body system in general form is first established; computational accuracy and computational efficiency of simulation and control of the flexible body system caused by different shape functions through the analysis of the coefficient matrix in the kinetic equation aiming at the shape functions in three forms. Finally, the simulation calculation is carried out for the kinetic equation using different types of deformation functions through an example of double connecting rod.

\section{Flexible Multi-body System Dynamics}

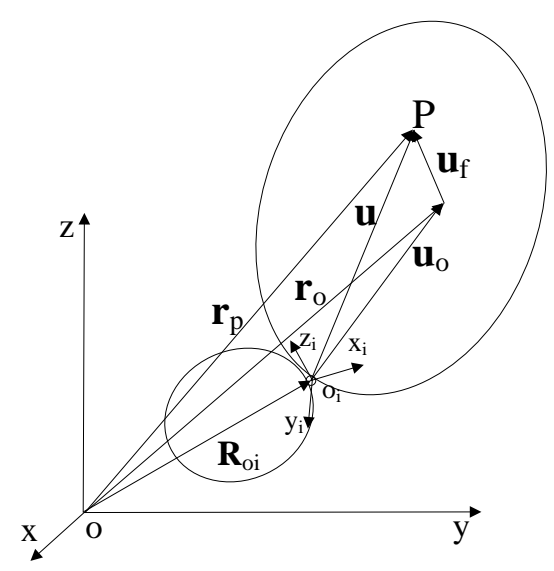

Fig.1 Finged flexible body

As shown in Figure 1, flexible bodies connected by any two hinges in the system are considered, and the coordinate system is established.The inertial coordinate system is located at $O$ point, and $O_{i} x_{i} y_{i} z_{i}$ is the floating coordinate system of the $i$ th object. The origin $O_{i}$ of the floating coordinate system is located at the hinge between the $i$-1th flexible body and the $i$ th flexible body, and its position vector in the inertial coordinate system is $\boldsymbol{R}_{O i}$. The ith object is taken as the research object, and the displacement of any point $\mathrm{P}$ on the flexible body is the sum of rigid motion $\boldsymbol{r}_{o}$ and elastic motion $\boldsymbol{u}_{f}$. In the moving coordinate system, setting $\boldsymbol{u}_{O}$ to the undeformed position vector, $\boldsymbol{u}_{f}$ is the position vector caused by the arbitrary deformation; setting $\boldsymbol{u}$ to the position vector of $\mathrm{P}$ point relative to the moving coordinate system, $\boldsymbol{u}=\boldsymbol{u}_{O}+\boldsymbol{u}_{f}$.

When any $\mathrm{P}$ point goes through the flexible point, the displacement of the flexible body forms a displacement field $\boldsymbol{\Phi}(x, y, z)$, and this displacement field is compatible and complete. In order to further describe the deformation mode of the object, setting the shape function matrix $\boldsymbol{\Phi}=\left[\begin{array}{llll}\boldsymbol{\Phi}_{1} & \boldsymbol{\Phi}_{2} & \cdots & \boldsymbol{\Phi}_{n}\end{array}\right]$, the amount of deformation of each point on the elastomer $\boldsymbol{u}_{f}$ can be expressed as $\boldsymbol{u}_{f}=\boldsymbol{\Phi} \boldsymbol{q}_{f}$, in which $\boldsymbol{q}_{f}$ indicates the generalized coordinates corresponding to the deformation. In the inertial coordinate system, the position vector of $\mathrm{P}$ point can be expressed as:

$$
\boldsymbol{r}_{p}=\boldsymbol{R}_{O i}+\boldsymbol{A} \boldsymbol{u}=\boldsymbol{R}_{O i}+\boldsymbol{A}\left(\boldsymbol{u}_{O}+\boldsymbol{u}_{f}\right)=\boldsymbol{R}_{O i}+\boldsymbol{A}\left(\boldsymbol{u}_{O}+\boldsymbol{\Phi} \boldsymbol{q}_{f}\right)
$$


In the formula, $\boldsymbol{A}$ indicates the rotation transformation matrix of the moving coordinate system relative to the inertial coordinate system. The derivative of Formula (1) is taken, to obtain the velocity vector of $\mathrm{P}$ point:

$$
\dot{\boldsymbol{r}}_{p}=\dot{\boldsymbol{R}}_{O i}+\dot{\boldsymbol{A}} \boldsymbol{u}+\boldsymbol{A} \dot{\boldsymbol{u}}=\dot{\boldsymbol{R}}_{O i}+\dot{\boldsymbol{A}} \boldsymbol{u}+\boldsymbol{A} \boldsymbol{\Phi} \dot{\boldsymbol{q}}_{f}
$$

From the definition of Euler parameters ${ }^{[15]}$, the rotation transformation matrix can be expressed as $\boldsymbol{A}=\hat{\boldsymbol{E}} \hat{\boldsymbol{G}}^{T} . \dot{\boldsymbol{A}} \boldsymbol{u}=-2 \boldsymbol{A} \tilde{\boldsymbol{u}} \hat{\boldsymbol{G}} \dot{\boldsymbol{P}}=\boldsymbol{B} \dot{\boldsymbol{P}}$ is set. The velocity vector of $\mathrm{P}$ point can be expressed as the matrix form.

$$
\dot{\boldsymbol{r}}_{p}=\left[\begin{array}{lll}
\boldsymbol{I} & \boldsymbol{B} & \boldsymbol{A} \boldsymbol{\Phi}
\end{array}\right]\left[\begin{array}{c}
\dot{\boldsymbol{R}}_{O i} \\
\dot{\boldsymbol{P}} \\
\dot{\boldsymbol{q}}_{f}
\end{array}\right]
$$

In Formula (2), the velocity vector of any point on any flexible body is determined, and it shows that the shape function affects velocity vector and kinetic energy of any point on the flexible body, and the selection of the shape function is crucial to the solution of the equation of the flexible body system dynamics.

\section{Basic Equations of Dynamics}

From the expression of velocity vector of any $P$ point, the kinetic energy of the flexible body $i$ can be obtained:

$$
T=\frac{1}{2} \int_{v} \rho \dot{\boldsymbol{r}}_{p}^{T} \dot{\boldsymbol{r}}_{p} d V=\frac{1}{2} \dot{\boldsymbol{q}}^{T} \boldsymbol{M} \dot{\boldsymbol{q}}
$$

Calculation of potential energy of flexible body. The virtual work of the internal force caused by the elastic deformation is:

$$
\begin{aligned}
\delta W & =-\int_{v} \boldsymbol{\sigma}^{T} \delta \boldsymbol{\varepsilon} \mathrm{d} V \\
& =-\int_{v} \boldsymbol{\varepsilon}^{T} \boldsymbol{E}^{T} \delta \boldsymbol{\varepsilon} \mathrm{d} V \\
& =-\int_{v} \boldsymbol{q}_{f}^{T}\left(\boldsymbol{D}^{*} \boldsymbol{\Phi}\right)^{T} \boldsymbol{E} \boldsymbol{D}^{*} \boldsymbol{\Phi} \delta \boldsymbol{q}_{f} \mathrm{~d} V \\
& =-\boldsymbol{q}_{f}^{T}\left[\int_{v}\left(\boldsymbol{D}^{*} \boldsymbol{\Phi}\right)^{T} \boldsymbol{E} \boldsymbol{D}^{*} \boldsymbol{\Phi} \mathrm{d} V\right] \delta \boldsymbol{q}_{f} \\
& =-\boldsymbol{q}_{f}^{T} \boldsymbol{K}_{f f} \delta \boldsymbol{q}_{f} \\
& =\delta U
\end{aligned}
$$

In the formula, $\boldsymbol{\varepsilon}=\boldsymbol{D}^{*} \boldsymbol{u}_{f}=\boldsymbol{D}^{*} \boldsymbol{\Phi} \boldsymbol{q}_{f} ; \boldsymbol{\sigma}=\boldsymbol{E} \boldsymbol{\varepsilon}=\boldsymbol{E} \boldsymbol{D}^{*} \boldsymbol{\Phi} \boldsymbol{q}_{f} ; \boldsymbol{D}^{*}$ indicates the differential operator matrix; $\boldsymbol{E}$ indicates the elastic modulus matrix. $\boldsymbol{Q}_{e}=-\boldsymbol{q}_{f}^{T} \boldsymbol{K}$ indicates the generalized force of the elastic force caused by the deformation of the elastomer; $\boldsymbol{K}_{f f}=\int_{v}\left(\boldsymbol{D}^{*} \boldsymbol{\Phi}\right)^{T} \boldsymbol{E} \boldsymbol{D}^{*} \boldsymbol{\Phi} \mathrm{d} V$ indicates the stiffness matrix of the elastomer corresponding to generalized coordinates $\boldsymbol{q}_{f}$, which is the symmetric positive definite matrix. The strain energy of the elastomer is:

$$
U=\frac{1}{2} \boldsymbol{q}_{f}^{T} \boldsymbol{K}_{f f} \boldsymbol{q}_{f}=\frac{1}{2} \boldsymbol{q}^{T} \boldsymbol{K} \boldsymbol{q}
$$

Calculation of generalized force. Setting the concentrated force to $\boldsymbol{F}=\boldsymbol{F}(\boldsymbol{q}, t)$ acting on any point on the flexible body, the virtual work on the virtual displacement of its action point is:

$$
\delta W=\boldsymbol{F}^{T}\left[\begin{array}{lll}
\boldsymbol{I} & \boldsymbol{B} & \boldsymbol{A} \boldsymbol{\Phi}
\end{array}\right]\left[\begin{array}{c}
\delta \boldsymbol{R}_{O i} \\
\delta \boldsymbol{P} \\
\delta \boldsymbol{q}_{f}
\end{array}\right]=\left[\begin{array}{lll}
\boldsymbol{Q}_{F R}^{T} & \boldsymbol{Q}_{F \theta}^{T} & \boldsymbol{Q}_{F f}^{T}
\end{array}\right]\left[\begin{array}{c}
\delta \boldsymbol{R}_{O i} \\
\delta \boldsymbol{P} \\
\delta \boldsymbol{q}_{f}
\end{array}\right]
$$

The generalized force corresponding to the active force $\boldsymbol{F}$ is: 


$$
\boldsymbol{Q}_{F}^{T}=\left[\begin{array}{lll}
\boldsymbol{Q}_{F R}^{T} & \boldsymbol{Q}_{F \theta}^{T} & \boldsymbol{Q}_{F f}^{T}
\end{array}\right]=\boldsymbol{F}^{T}\left[\begin{array}{lll}
\boldsymbol{I} & \boldsymbol{B} & \boldsymbol{A} \boldsymbol{\Phi}
\end{array}\right]
$$

From the Lagrange equation $\frac{\mathrm{d}}{\mathrm{d} t}\left(\frac{\partial T}{\partial \dot{\boldsymbol{q}}}\right)-\frac{\partial T}{\partial \boldsymbol{q}}+\frac{\partial U}{\partial \boldsymbol{q}}=\boldsymbol{Q}$, the equation of flexible body dynamics can be deduced:

$$
\left[\begin{array}{ccc}
\boldsymbol{m}_{R R} & \boldsymbol{m}_{R \theta} & \boldsymbol{m}_{R f} \\
\cdots & \boldsymbol{m}_{\theta \theta} & \boldsymbol{m}_{\theta f} \\
\cdots & \cdots & \boldsymbol{m}_{f f}
\end{array}\right]\left[\begin{array}{c}
\ddot{\boldsymbol{R}}_{O i} \\
\ddot{\boldsymbol{P}} \\
\ddot{\boldsymbol{q}}_{f}
\end{array}\right]+\left[\begin{array}{ccc}
0 & 0 & 0 \\
0 & 0 & 0 \\
0 & 0 & \boldsymbol{K}_{f f}
\end{array}\right]\left[\begin{array}{c}
\boldsymbol{R}_{O i} \\
\boldsymbol{P} \\
\boldsymbol{q}_{f}
\end{array}\right]=\left[\begin{array}{c}
\boldsymbol{Q}_{F R} \\
\boldsymbol{Q}_{F \theta} \\
\boldsymbol{Q}_{F f}
\end{array}\right]+\left[\begin{array}{c}
\boldsymbol{Q}_{v R} \\
\boldsymbol{Q}_{v \theta} \\
\boldsymbol{Q}_{v f}
\end{array}\right]
$$

In the formula, $\boldsymbol{Q}_{v}=\left[\begin{array}{lll}\boldsymbol{Q}_{v R} & \boldsymbol{Q}_{v \theta} & \boldsymbol{Q}_{v f}\end{array}\right]^{T}=-\dot{\boldsymbol{M}} \dot{\boldsymbol{q}}+\frac{\partial}{\partial \boldsymbol{q}}\left(\frac{1}{2} \dot{\boldsymbol{q}}^{T} \boldsymbol{M} \dot{\boldsymbol{q}}\right)$, The expression of each element of mass matrix is:

$$
\boldsymbol{M}=\left[\begin{array}{ccc}
\boldsymbol{m}_{R R} & \boldsymbol{m}_{R \theta} & \boldsymbol{m}_{R f} \\
\cdots & \boldsymbol{m}_{\theta \theta} & \boldsymbol{m}_{\theta f} \\
\cdots & \cdots & \boldsymbol{m}_{f f}
\end{array}\right]=\int_{v} \rho\left[\begin{array}{ccc}
\boldsymbol{I} & \boldsymbol{B} & \boldsymbol{A} \boldsymbol{\Phi} \\
\boldsymbol{B}^{T} & \boldsymbol{B}^{T} \boldsymbol{B} & \boldsymbol{B}^{T} \boldsymbol{A} \boldsymbol{\Phi} \\
\boldsymbol{\Phi}^{T} \boldsymbol{A} & \boldsymbol{\Phi}^{T} \boldsymbol{A} \boldsymbol{B} & \boldsymbol{\Phi}^{T} \boldsymbol{A}^{T} \boldsymbol{A} \boldsymbol{\Phi}
\end{array}\right] \mathrm{d} V
$$

It shows from the deduction of the expressions of mass matrix $\boldsymbol{M}$ and stiffness matrix $\boldsymbol{K}$ the kinetic equation of the flexible body system that under the specified floating coordinate system, the form of the kinetic equation of the flexible body system and various coefficient matrix elements are same, thus the decoupling degree of the equation is same, which shows that the selection of the shape function has little to do with the decoupling degree of the equation. Different forms of shape function result in coefficient matrix of different complexity and different amount of generalized coordinates, thus the shape function affects computational accuracy and efficiency of the kinetic equation. According to the assumption method used at present, although the solution for the problem can be obtained, the estimation of its accuracy is very difficult, and the error is time-varying function, which is just the difficulty of the flexible body dynamics.

\section{Shape Function of Flexible Body}

The configuration of the flexible body in the motion process is changing. By means of discretization, the relative deformation of the flexible body is expressed as $\mathrm{N}$ linear combinations of generalized coordinates $\boldsymbol{q}_{i}(i=1, \cdots, N)$, and the expression of the general displacement of the deformation is:

$$
\boldsymbol{u}_{f}=\sum_{i=1}^{N} \Phi_{i} q_{i}=\boldsymbol{\Phi} \boldsymbol{q}_{f}
$$

In the formula, $\boldsymbol{\Phi}$ indicates the shape function in general form, which is only related to the position. Assuming that the suitable shape function is the core problem of discretization, the selected shape function is expected to describe the actual deformation of the object as much as possible. Static deformation, finite element model and vibration modal are three main types of shape functions.

Considering double-connecting rod mechanism shown in Figure 2, $m_{1}$ and $m_{2}$ indicate the lumped mass at the terminal, and two connecting rods are uniform Euler beams, whose mass is $M_{1}$ and $M_{2}$, length is $l_{1}$ and $l_{2}$, and stiffness is $E I_{1}$ and $E I_{2}$ respectively. The deformation of the connecting rod is described with three types of shape functions below. 


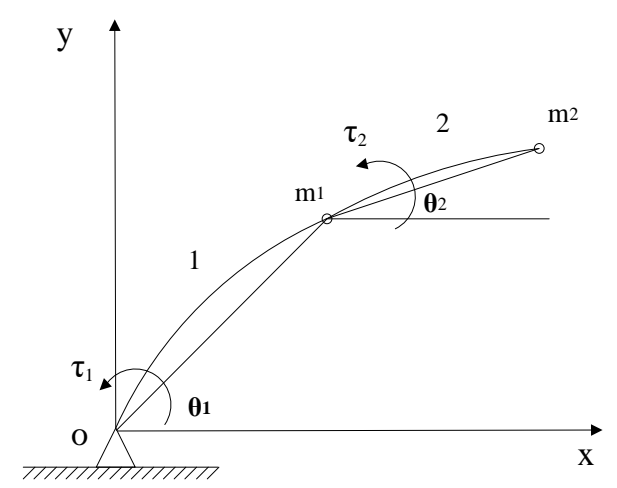

Fig.2 Dual-link planar motion mechanism

Case (1): The static deformation is taken as the shape function. The static deformation model is to take the static deformation of the object under the external force including the gravity as its assumed deformation mode. The connecting rod can be equivalent to the cantilever beam model, affected by the self-weight and the concentrated force at the terminal. From the knowledge of mechanics of material, the flexibility of connecting rod affected by the uniform load (self-weight) and the concentrated force at the terminal is respectively:

$$
\delta_{1}=-\frac{x^{2}}{6 E_{1} I}(3 l-x) \quad \delta_{2}=-\frac{x^{2}}{24 E_{1} I l}\left(x^{2}-4 l x+6 l^{2}\right)
$$

The amounts of deformation of connecting rod 1 and connecting rod 2 are respectively expressed as:

$$
u_{f 1}=\delta_{1} m_{1} g+\delta_{2} M_{1} g \quad u_{f 2}=\delta_{1} m_{2} g+\delta_{2} M_{2} g
$$

The elastic deformation in the motion of the flexible body is described with the static deformation model, and the accuracy increases compared with the calculation with the rigid body model. The static deformation model is simple, the needed number of generalized coordinates is small, and the computational efficiency is high. However, if the elastic deformation of the flexible body is described by the static deformation instead of the dynamic deformation, the computational accuracy must have large error.

Case (2): The finite element model is taken as the shape function. The finite element method is to break up the whole into parts of the object with complex shape, boundary condition and load, which is segmented to the elements of limited number, limited size and certain regular shape. The displacement vector $\boldsymbol{u}_{f}^{i j}$ at any point $\mathrm{P}$ on the $j$ element of $i$ object can be expressed as:

$$
\boldsymbol{u}_{f}^{i j}=\boldsymbol{N}^{i j} \boldsymbol{q}_{f}^{i}
$$

In the formula, $\boldsymbol{N}^{i j}$ indicates the deformation mode or the assumed displacement field of $j$ element, which is called the shape function of $j$ element, and $\boldsymbol{q}_{f}^{i}$ indicates the displacement vector of the node of this element. After assembly of all the elements, the displacement vector of all the nodes of the object constitute the elastic generalized coordinates of this object. In the model shown in Figure 2, the connecting rod is equivalent to the beam model and divided into $i$ elements, and the length of the element is $l$. The displacement of element node is deflection and rotation angle of the node.

$$
\boldsymbol{q}_{f}^{i}=\left[\begin{array}{llll}
w_{i} & \theta_{i} & w_{i+1} & \theta_{i+1}
\end{array}\right]
$$

Through the polynomial fitting, the shape function expressed by the node displacement of the element can be obtained:

$$
N^{i j}=\left[\begin{array}{llll}
1-3 \xi^{2}+2 \xi^{3} & l\left(\xi-2 \xi^{2}+3 \xi^{3}\right) & 3 \xi^{2}-2 \xi^{3} & l\left(-\xi^{2}+\xi^{3}\right)
\end{array}\right]
$$

The derivative of the above formula is taken, to obtain the geometric matrix of the beam element:

$$
\boldsymbol{B}^{i j}=\left(\boldsymbol{N}^{i j}\right)^{\prime \prime}=\frac{1}{l^{2}}[-6+12 \xi \quad l(-4+6 \xi) \quad 6-12 \xi \quad l(-2+6 \xi)]
$$

The expression of elastic potential energy of Bernoulli beam is: 


$$
U_{i j}=\frac{1}{2} \int_{0}^{l} E I\left(u_{f}^{i j}\right)^{2} \mathrm{~d} x=\frac{1}{2} \boldsymbol{q}_{f}^{i T}\left[\int_{0}^{l} \boldsymbol{B}^{T} E I \boldsymbol{B}\right] \boldsymbol{q}_{f}^{i}=\frac{1}{2} \boldsymbol{q}_{f}^{i T} \boldsymbol{K}^{i j} \boldsymbol{q}_{f}^{i}
$$

The stiffness matrix of the element can be obtained:

$$
\boldsymbol{K}^{i j}=\int_{0}^{l} \boldsymbol{B}^{T} E I \boldsymbol{B}=\frac{E I}{l^{3}}\left[\begin{array}{cccc}
12 & 6 l & -12 & 6 l \\
\ldots & 4 l^{2} & -6 l & 2 l^{2} \\
\ldots & \ldots & 12 & -6 l \\
\ldots & \ldots & \ldots & 4 l^{2}
\end{array}\right]
$$

The consistent mass matrix of the element can be obtained according to the Literature ${ }^{[16]}$ :

$$
\boldsymbol{M}^{i j}=\frac{\rho A l}{420}\left[\begin{array}{cccc}
156 & 22 l & 54 & -13 l \\
\cdots & 4 l^{2} & 13 l & -3 l \\
\cdots & \cdots & 156 & -22 l \\
\cdots & \cdots & \cdots & 4 l^{2}
\end{array}\right]
$$

After stiffness matrix and mass matrix of the beam element are obtained, stiffness matrix and mass matrix of the system can be assembled according to the initial condition, and the vibration analysis is carried out then. In the calculation of the finite element model, the selection of the deformation mode $\boldsymbol{N}^{i j}$ of the element has multiple forms, which can be selected according to factors such as materials and shapes as well as computational accuracy and efficiency limitation of the flexible body as appropriate. If the number of divided elements is more, the number of generalized coordinates is more, and computational accuracy is higher; and the computational efficiency decreases subsequently.

Case (3): The vibration modal is taken as the shape function. The vibration modal is a kind of method commonly used that describes the spatial deformation of the object in the structural dynamics. It describes the deformation of any point on the flexible body by introducing modal vector and mode coordinates, namely:

$$
\boldsymbol{u}_{f}=\boldsymbol{\Phi} \boldsymbol{q}_{f}
$$

In the formula, $\boldsymbol{\Phi}=\left[\begin{array}{llll}\boldsymbol{\Phi}_{1} & \boldsymbol{\Phi}_{2} & \cdots & \boldsymbol{\Phi}_{N}\end{array}\right]$ indicates the modal vector matrix; $\boldsymbol{q}_{f}=\boldsymbol{q}_{f}(t)$ indicates the mode coordinates; and $N$ indicates the number of mode truncations. In the model shown in Figure 2, the deformations of connecting rod 1 and connecting rod 2 are respectively:

$$
u_{f 1}=\sum_{i=1}^{N} q_{1 i}(t) \varphi_{i}\left(x_{1}\right), \quad u_{f 2}=\sum_{i=1}^{N} q_{2 i}(t) \varphi_{i}\left(x_{2}\right)
$$

In them, $\varphi_{i}(x)=\sin \frac{i \pi x}{l}$ indicates the $i$-order vibration mode of the beam, and $q_{i}(t)$ indicates the mode coordinates.

The range of modal truncation can be considered according to transcendental response characteristics and accuracy requirement. In general, the response contribution of the low-order modal is large, and the response contribution of the high-order modal is relatively small. Under the premise that the solving accuracy is guaranteed, the modal with small contributions can be truncated, to reduce the solving scale to the utmost.

\section{Case Study}

Setting $\mathbf{r}_{1}$ and $\mathbf{r}_{2}$ to position vectors of point $P_{1} 1$ and $P_{2} 2$ on connecting rod 1 and connecting rod 2 in the inertial coordinate system, the kinetic energy of the system is:

$$
T=T_{1}+T_{2}=\int_{l_{1}} \rho \dot{r}_{1}^{T} \dot{r}_{1} d l_{1}+\int_{l_{2}} \rho \dot{\boldsymbol{r}}_{2}^{T} \dot{\boldsymbol{r}}_{2} d l_{2}
$$

Calculation of the kinetic energy of connecting rod 1. 


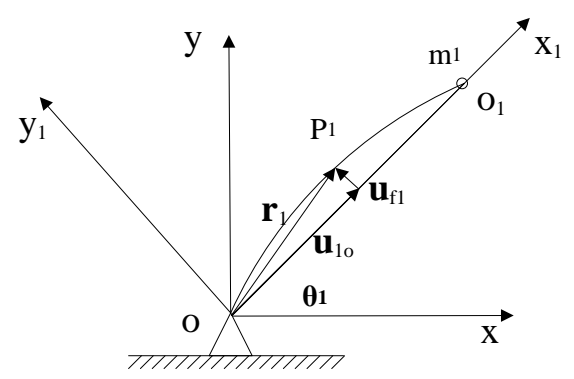

Fig. 3 Icon of link 1

The selection of the coordinate system is shown in Figure 3, and the position vector $\boldsymbol{r}_{1}$ of any point $\mathrm{P}_{1}$ on connecting rod 1 can be obtained:

$$
\boldsymbol{r}_{1}=\boldsymbol{A}_{1}\left(\boldsymbol{u}_{1 O}+\boldsymbol{u}_{f 1}\right)
$$

The derivative of Formula (22) is taken to obtain the velocity vector $\dot{\mathbf{r}}_{1}$ of $P_{1}$ :

$$
\dot{\boldsymbol{r}}_{1}=\boldsymbol{A}_{1}\left(\dot{\boldsymbol{u}}_{1 O}+\dot{\boldsymbol{u}}_{f 1}\right)+\dot{\boldsymbol{A}}_{1}\left(\boldsymbol{u}_{1 O}+\boldsymbol{u}_{f 1}\right)=\boldsymbol{A}_{1}\left[\dot{\boldsymbol{u}}_{f 1}+\boldsymbol{A}_{1}^{T} \dot{\boldsymbol{A}}_{1}\left(\boldsymbol{u}_{1 O}+\boldsymbol{u}_{f 1}\right)\right]=\boldsymbol{A}_{1}\left[\dot{\boldsymbol{u}}_{f 1}+\boldsymbol{\omega}_{1}\left(\boldsymbol{u}_{1 O}+\boldsymbol{u}_{f 1}\right)\right]
$$

In them, $\boldsymbol{A}_{1}=\left[\begin{array}{cc}\cos \theta_{1} & -\sin \theta_{1} \\ \sin \theta_{1} & \cos \theta_{1}\end{array}\right], \quad \boldsymbol{\omega}_{1}=\boldsymbol{A}_{1}^{T} \dot{\boldsymbol{A}}_{1}, \quad \boldsymbol{u}_{1 O}=\left[\begin{array}{c}x_{1} \\ 0\end{array}\right], \quad \boldsymbol{u}_{f 1}=\left[\begin{array}{c}0 \\ v_{1}\end{array}\right], \quad \dot{\boldsymbol{u}}_{1 O}=0$

The kinetic energy of connecting rod 1 is:

$$
\begin{aligned}
T_{1} & =\frac{1}{2} \int_{0}^{l_{1}} \rho_{1}\left(x_{1}\right) \dot{\boldsymbol{r}}_{1}^{T} \dot{\boldsymbol{r}}_{1} \mathrm{~d} x_{1} \\
& =\frac{1}{2} \int_{0}^{l_{1}} \rho_{1}\left(x_{1}\right)\left[\dot{\boldsymbol{u}}_{f 1}^{T} \dot{\boldsymbol{u}}_{f 1}+2 \dot{\boldsymbol{u}}_{f 1}^{T} \boldsymbol{\omega}_{1}\left(\boldsymbol{u}_{1 O}+\boldsymbol{u}_{f 1}\right)+\left(\boldsymbol{u}_{1 O}+\boldsymbol{u}_{f 1}\right)^{T} \boldsymbol{\omega}_{1}^{T} \boldsymbol{\omega}_{1}\left(\boldsymbol{u}_{1 O}+\boldsymbol{u}_{f 1}\right)\right] \mathrm{d} x_{1} \\
& =\frac{1}{2} \int_{0}^{l_{1}} \rho_{1}\left(x_{1}\right)\left[\left(\frac{\partial v_{1}}{\partial t}\right)^{2}+2 x_{1} \dot{\theta}_{1} \frac{\partial v_{1}}{\partial t}+\left(x_{1}\right)^{2} \dot{\theta}_{1}^{2}+v_{1}^{2} \dot{\theta}_{1}^{2}\right] \mathrm{d} x_{1} \\
& =\frac{1}{2} m_{1} l_{1}^{2} \dot{\theta}_{1}^{2}+\frac{1}{6} M_{1} l_{1}^{2} \dot{\theta}_{1}^{2}+\frac{1}{2} \frac{M_{1}}{l_{1}} \int_{0}^{l_{1}}\left(\frac{\partial v_{1}}{\partial t}\right)^{2} \mathrm{~d} x_{1}+\frac{M_{1}}{l_{1}} \dot{\theta}_{1} \int_{0}^{l_{1}} x_{1} \frac{\partial v_{1}}{\partial t} \mathrm{~d} x_{1}+\frac{1}{2} \frac{M_{1}}{l_{1}} \dot{\theta}_{1}^{2} \int_{0}^{l_{1}} v_{1}^{2} \mathrm{~d} x_{1}
\end{aligned}
$$

In the formula, $v_{1}$ indicates the deflection of connecting rod 1 and the density of connecting rod 1 is: $\rho_{1}\left(x_{1}\right)=\frac{M_{1}}{l_{1}}+m_{1} \delta\left(x_{1}-l_{1}\right)$.

Calculation of kinetic energy of connecting rod 2.

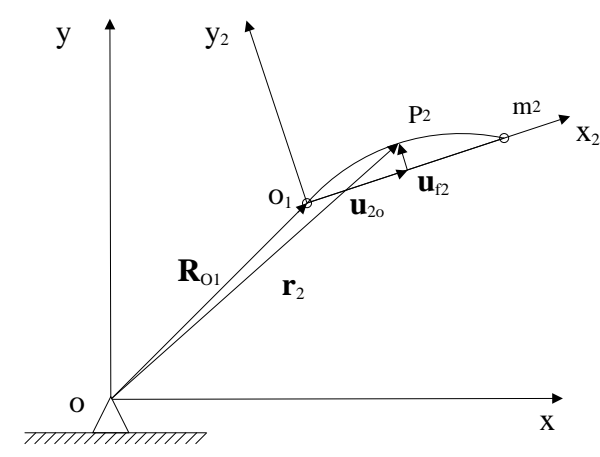

Fig. 4 Icon of link 2

The selection of the coordinate system is shown in Figure 4, and the position vector $\boldsymbol{r}_{2}$ of any point $\mathrm{P}_{2}$ of connecting rod 2 :

$$
\boldsymbol{r}_{2}=\boldsymbol{R}_{O 1}+\boldsymbol{A}_{2}\left(\boldsymbol{u}_{2 O}+\boldsymbol{u}_{f 2}\right)=\boldsymbol{A}_{1} \boldsymbol{R}_{O 1}^{\prime}+\boldsymbol{A}_{2}\left(\boldsymbol{u}_{2 O}+\boldsymbol{u}_{f 2}\right)
$$


In the formula, $\boldsymbol{R}_{O 1}^{\prime}$ indicates the position vector of the moving coordinate origin of connecting rod 2 in the moving coordinate system of connecting rod 1. The derivative of Formula (24) is taken, to obtain velocity vector $\dot{\boldsymbol{r}}_{2}$ of $\mathrm{P}_{2}$ :

$$
\dot{\boldsymbol{r}}_{2}=A_{1}\left(\dot{\boldsymbol{R}}_{O 1}^{\prime}+\boldsymbol{\omega}_{1} \boldsymbol{R}_{O 1}^{\prime}\right)+A_{2}\left[\dot{\boldsymbol{u}}_{f 2}+\boldsymbol{\omega}_{2}\left(\boldsymbol{u}_{2 O}+\boldsymbol{u}_{f 2}\right)\right]
$$

In them, $\boldsymbol{A}_{2}=\left[\begin{array}{cc}\cos \theta_{2} & -\sin \theta_{2} \\ \sin \theta_{2} & \cos \theta_{2}\end{array}\right], \boldsymbol{\omega}_{2}=\boldsymbol{A}_{2}^{T} \boldsymbol{A}_{2}, \dot{\boldsymbol{R}}_{O 1}^{\prime}=0, \boldsymbol{u}_{f 2}=\left[\begin{array}{c}0 \\ v_{2}\end{array}\right]$

The kinetic energy of connecting rod 2 is:

$$
\begin{aligned}
T_{2}= & \frac{1}{2} \int_{0}^{l_{2}} \rho_{2}\left(x_{2}\right) \dot{\mathbf{r}}_{2}^{T} \dot{\mathbf{r}}_{2} \mathrm{~d} x_{2} \\
= & \frac{1}{2} \int_{0}^{l_{2}} \rho_{2}\left(x_{2}\right)\left(\begin{array}{l}
\mathbf{R}_{O 1}^{\prime T} \boldsymbol{\omega}_{1}^{T} \boldsymbol{\omega}_{1} \mathbf{R}_{O 1}^{\prime}+\dot{\mathbf{u}}_{f 2}^{T} \dot{\mathbf{u}}_{f 2}+2 \dot{\mathbf{u}}_{f 2}^{T} \boldsymbol{\omega}_{2}\left(\mathbf{u}_{2 O}+\mathbf{u}_{f 2}\right)+\left(\mathbf{u}_{2 O}+\mathbf{u}_{f 2}\right)^{T} \boldsymbol{\omega}_{2}^{T} \boldsymbol{\omega}_{2}\left(\mathbf{u}_{2 O}+\mathbf{u}_{f 2}\right) \\
+2 \mathbf{R}_{O 1}^{\prime T} \boldsymbol{\omega}_{1}^{T} \mathbf{A}_{1}^{T} \mathbf{A}_{2} \dot{\mathbf{u}}_{f 2}+2 \mathbf{R}_{O 1}^{\prime T} \boldsymbol{\omega}_{1}^{T} \mathbf{A}_{1}^{T} \mathbf{A}_{2} \boldsymbol{\omega}_{2}\left(\mathbf{u}_{2 O}+\mathbf{u}_{f 2}\right)
\end{array}\right) \mathrm{d} x_{2} \\
= & \frac{1}{2} m_{2} l_{1}^{2} \dot{\theta}_{1}^{2}+\frac{1}{2} m_{2} l_{2}^{2} \dot{\theta}_{2}^{2}+m_{2} l_{1} l_{2} \dot{\theta}_{1} \dot{\theta}_{2} \cos \left(\theta_{1}-\theta_{2}\right) \\
& +\frac{1}{2} M_{2} l_{1}^{2} \dot{\theta}_{1}^{2}+\frac{1}{6} M_{2} l_{2}^{2} \dot{\theta}_{2}^{2}+\frac{1}{2} M_{2} l_{1} l_{2} \dot{\theta}_{1} \dot{\theta}_{2} \cos \left(\theta_{1}-\theta_{2}\right) \\
& +\frac{1}{2} \frac{M_{2}}{l_{2}} \int_{0}^{l_{2}}\left(\frac{\partial v_{2}}{\partial t}\right)^{2} \mathrm{~d} x_{2}+M_{2} \frac{l_{1}}{l_{2}} \dot{\theta}_{1} \cos \left(\theta_{1}-\theta_{2}\right) \int_{0}^{l_{2}} \frac{\partial v_{2}}{\partial t} \mathrm{~d} x_{2} \\
& +\frac{1}{2} \frac{M_{2}}{l_{2}} \dot{\theta}_{2}^{2} \int_{0}^{l_{2}} v_{2}^{2} \mathrm{~d} x_{2}+\frac{M_{2}}{l_{2}} \dot{\theta}_{2} \int_{0}^{l_{2}} x_{2} \frac{\partial v_{2}}{\partial t} \mathrm{~d} x_{2} \\
& +M_{2} \frac{l_{1}}{l_{2}} \dot{\theta}_{1} \dot{\theta}_{2} \sin \left(\theta_{1}-\theta_{2}\right) \int_{0}^{l_{2}} v_{2} \mathrm{~d} x_{2}
\end{aligned}
$$

In the formula, $v_{2}$ indicates the deflection of connecting rod 2, and the mass density of connecting rod 2 is $\rho_{2}\left(x_{2}\right)=\frac{M_{2}}{l_{2}}+m_{2} \delta\left(x_{2}-l_{2}\right)$.

Case (1): Deduced with the static correction model, Formula (12), Formula (24), and Formula (27) are substituted into Lagrange equation, to obtain the expression of the nonzero element in the mass matrix $\boldsymbol{M}$ :

$$
\begin{aligned}
& m_{11}=\left(m_{1}+m_{2}+\frac{M_{1}}{3}+M_{2}\right) l_{1}^{2}+\frac{M_{1}}{l_{1}} \int_{0}^{l_{1}} u_{f 1}^{2} \mathrm{~d} x_{1} \quad m_{22}=\left(m_{2}+\frac{M_{2}}{3}\right) l_{2}^{2}+\frac{M_{2}}{l_{2}} \int_{0}^{l_{2}} u_{f 2}^{2} \mathrm{~d} x_{2} \\
& m_{12}=m_{21}=\left(m_{2}+\frac{1}{2} M_{2}\right) \cos \left(\theta_{1}-\theta_{2}\right)+\frac{M_{2} l_{1}}{l_{2}} \sin \left(\theta_{1}-\theta_{2}\right) \int_{0}^{l_{2}} u_{f 2} \mathrm{~d} x_{2}
\end{aligned}
$$

Because the dynamic deformation of the rod is replaced by the static deformation, the generalized coordinates is not added in the system, and stiffness matrix $\boldsymbol{K}=\mathbf{0}$. The gravity potential energy of double connecting rod system is:

$$
\begin{aligned}
U_{g}= & \left(m_{1}+m_{2}+\frac{M_{1}}{2}+M_{2}\right) g l_{1} \sin \theta_{1}+\left(m_{2}+\frac{M_{2}}{2}\right) g l_{2} \sin \theta_{2} \\
& +\frac{M_{1} l_{1}^{3}\left(2 M_{1}+5 m_{1}\right)}{40 E I_{1}} g \cos \theta_{1}+\frac{M_{2} l_{2}^{3}\left(2 M_{2}+5 m_{2}\right)}{40 E I_{2}} g \cos \theta_{2}
\end{aligned}
$$

The driving torques $\tau_{1}(t)$ and $\tau_{2}(t)$ of double connecting rod system are respectively active forces of connecting rod 1 and connecting rod 2, and the corresponding generalized force can be obtained with the principle of virtual work:

$$
Q_{1}(t)=\tau_{1}(t)-\tau_{2}(t) \quad Q_{5}(t)=\tau_{2}(t)
$$

Case (2): The mass matrix and stiffness matrix of the element in the finite element model are deduced with Formula (18) and Formula (19), and mass matrix $\boldsymbol{M}$ and stiffness matrix $\boldsymbol{K}$ of the 
element are assembled, thus able to obtain mass matrix and stiffness matrix of the system, which is not described here.

Case (3): Deduced with the vibration modal, setting the number of mode truncations $n=3$, the function of the prior third-order vibration mode of the rod is:

$$
\varphi_{1}(x)=\sin \frac{\pi x}{l} \quad \varphi_{2}(x)=\sin \frac{2 \pi x}{l} \quad \varphi_{3}(x)=\sin \frac{3 \pi x}{l}
$$

Formula (20), Formula (23), Formula (26) and Formula (28) are substituted into Lagrange equation, to obtain the expression of the nonzero element in the mass matrix $\boldsymbol{M}$ :

$$
\begin{aligned}
& m_{11}=\left(m_{1}+m_{2}+\frac{M_{1}}{3}+M_{2}\right) l_{1}^{2}+\frac{M_{1}}{2}\left(a_{1}^{2}+a_{2}^{2}+a_{3}^{2}\right) \quad m_{22}=m_{33}=m_{44}=\frac{M_{1}}{2} \\
& m_{55}=\left(m_{2}+\frac{M_{2}}{3}\right) l_{2}^{2}+\frac{M_{2}}{2}\left(b_{1}^{2}+b_{2}^{2}+b_{3}^{2}\right) \quad m_{66}=m_{77}=m_{88}=\frac{M_{2}}{2} \\
& m_{12}=m_{21}=\frac{M_{1} l_{1}}{\pi} \quad m_{13}=m_{31}=-\frac{M_{1} l_{1}}{2 \pi} \quad m_{14}=m_{41}=\frac{M_{1} l_{1}}{3 \pi} \\
& m_{15}=m_{51}=\left(m_{2}+\frac{M_{2}}{2}\right) l_{1} l_{2} \cos \left(\theta_{1}-\theta_{2}\right)+2 \frac{M_{2} l_{1}}{3 \pi}\left(3 b_{1}+b_{3}\right) \sin \left(\theta_{1}-\theta_{2}\right) \\
& m_{16}=m_{61}=\frac{2 M_{2} l_{1}}{\pi} \cos \left(\theta_{1}-\theta_{2}\right) \quad m_{17}=m_{71}=0 \quad m_{18}=m_{81}=\frac{2 M_{2} l_{1}}{3 \pi} \cos \left(\theta_{1}-\theta_{2}\right) \\
& m_{56}=m_{65}=\frac{M_{2} l_{2}}{\pi} \quad m_{57}=m_{75}=-\frac{M_{2} l_{2}}{2 \pi} \quad m_{58}=m_{85}=\frac{M_{2} l_{2}}{3 \pi}
\end{aligned}
$$

The nonzero element in stiffness matrix $K$ is:

$$
\begin{array}{lll}
K_{22}=\frac{\pi^{4}}{2 l_{1}^{3}} E I_{1} & K_{33}=\frac{8 \pi^{4}}{l_{1}^{3}} E I_{1} & K_{44}=\frac{81 \pi^{4}}{2 l_{1}^{3}} E I_{1} \\
K_{66}=\frac{\pi^{4}}{2 l_{2}^{3}} E I_{2} & K_{77}=\frac{8 \pi^{4}}{l_{2}^{3}} E I_{2} & K_{88}=\frac{81 \pi^{4}}{2 l_{1}^{3}} E I_{2}
\end{array}
$$

The gravity potential energy of double connecting rod system is:

$$
\begin{aligned}
U_{g} & =\left(m_{1}+m_{2}+\frac{M_{1}}{2}+M_{2}\right) g l_{1} \sin \theta_{1}+\left(m_{2}+\frac{M_{2}}{2}\right) g l_{2} \sin \theta_{2} \\
& +\frac{2 M_{1} g}{3 \pi}\left(3 a_{1}+a_{3}\right) \cos \theta_{1}+\frac{2 M_{2} g}{3 \pi}\left(3 b_{1}+b_{3}\right) \cos \theta_{2}
\end{aligned}
$$

The driving torques $\tau_{1}(t)$ and $\tau_{2}(t)$ of double connecting rod system are respectively active forces of connecting rod 1 and connecting rod 2, and the corresponding generalized force can be obtained with the principle of virtual work:

$$
\begin{array}{llll}
Q_{1}(t)=\tau_{1}(t)-\tau_{2}(t) & Q_{2}(t)=\frac{\pi}{l_{1}}\left[\tau_{1}(t)-\tau_{2}(t)\right] & Q_{3}(t)=\frac{2 \pi}{l_{1}}\left[\tau_{1}(t)-\tau_{2}(t)\right] & Q_{4}(t)=\frac{3 \pi}{l_{1}}\left[\tau_{1}(t)-\tau_{2}(t)\right] \\
Q_{5}(t)=\tau_{2}(t) & Q_{6}(t)=\frac{\pi}{l_{2}} \tau_{2}(t) & Q_{7}(t)=\frac{2 \pi}{l_{2}} \tau_{2}(t) & Q_{8}(t)=\frac{3 \pi}{l_{2}} \tau_{2}(t)
\end{array}
$$

The numerical simulation can be carried out with static deformation model, finite element model and vibration modal respectively, and the simulation parameter is below:

$$
\begin{aligned}
& l_{1}=l_{2}=0.75 m \quad A_{1}=60 \times 15 \times 10^{-6} \mathrm{~m}^{2} \quad A_{2}=40 \times 10 \times 10^{-6} \mathrm{~m}^{2} \quad M_{1}=1.4 \mathrm{~kg} \quad M_{2}=0.62 \mathrm{~kg} \\
& E I_{1}=1220 \mathrm{~N} \cdot \mathrm{m}^{2} \quad E I_{2}=218 \mathrm{~N} \cdot \mathrm{m}^{2} \quad m_{1}=5.5 \mathrm{~kg} \quad m_{2}=0.62 \mathrm{~kg} \\
& \tau_{1}(t)=215 \sin ^{3}(2 \pi t)+62 N \cdot m \quad \tau_{2}(t)=75 \sin ^{3}(2 \pi t)+15 N \cdot m
\end{aligned}
$$

Assuming that two rods are in horizontal position at the initial moment, and the deformation is zero, the initial condition is $\boldsymbol{q}(0)=\mathbf{0}, \dot{\boldsymbol{q}}(0)=\mathbf{0}$ 
The prior third-order vibration modal is taken as reference, angular velocity and terminal displacement of the connecting rod as well as the error between them are calculated with static deformation model, first-order vibration mode, finite element model and rigid body model respectively, and the simulation result is below:
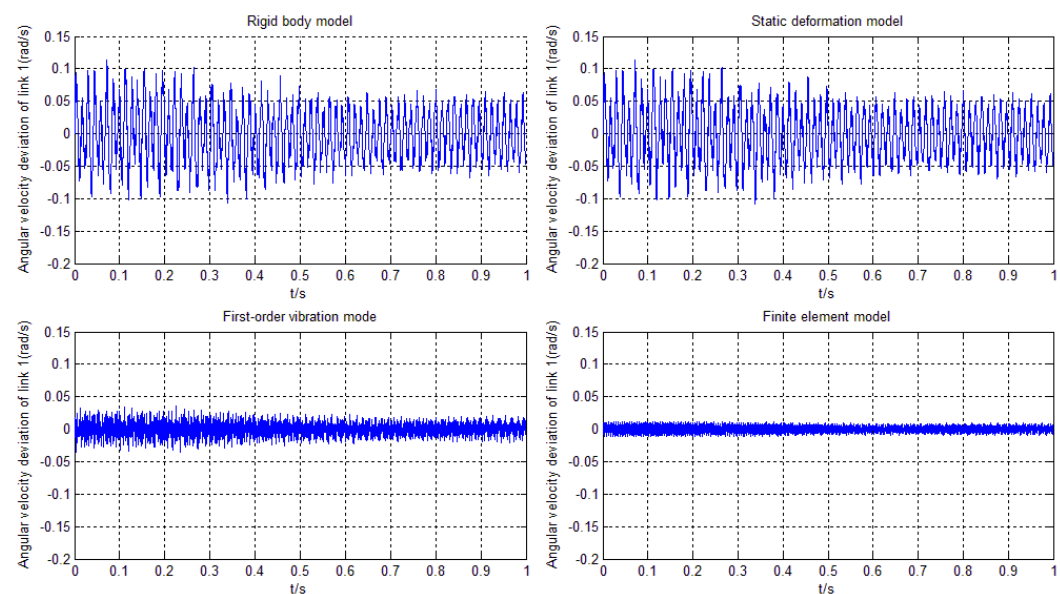

Fig.5 Angular velocity deviation of link 1
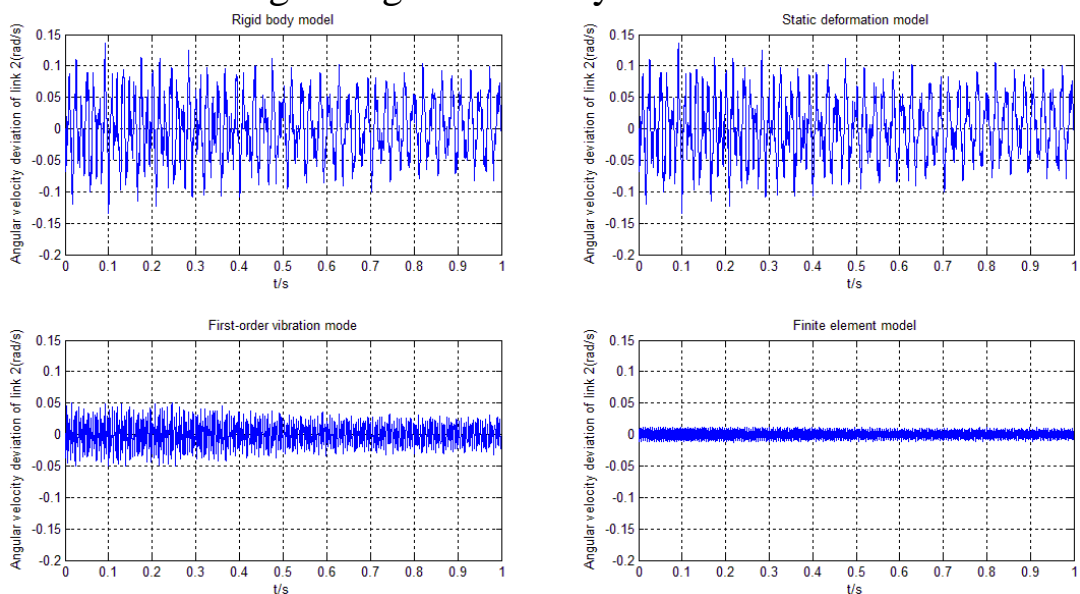

Fig.6 Angular velocity deviation of link 2

Figure 5 and Figure 6 show the error of angular velocity of connecting rod in rigid body model and flexible body model under three types of shape function. It shows that because it is in the static deformation model, the static deformation is taken as the shape function of the flexible body instead of the dynamic deformation, thus static deformation and rigid body model have the same influencing degree on the angular velocity of the flexible body. The error between rigid body model and static deformation is large, reaching $\pm 0.14 \mathrm{rad} / \mathrm{s}$, and the flexible deformation affects the rigid rotation angle of the rod. In the motion machinery with high accuracy requirement, the flexible deformation of the structure cannot be ignored. The calculation error of the first-order vibration mode is less than $\pm 0.05 \mathrm{rad} / \mathrm{s}$, and the computational accuracy increases significantly. For the general mechanical structure, under the premise that the solving accuracy is guaranteed, the high-order modal can be truncated, to reduce the solving scale to the utmost. The error of the finite element model is less than $\pm 0.02 \mathrm{rad} / \mathrm{s}$, and the computational accuracy is the maximum. However, because each element corresponds to multiple generalized coordinates, the coefficient matrix dimension of the kinetic equation of the system is high, and the computational efficiency is the minimum. 

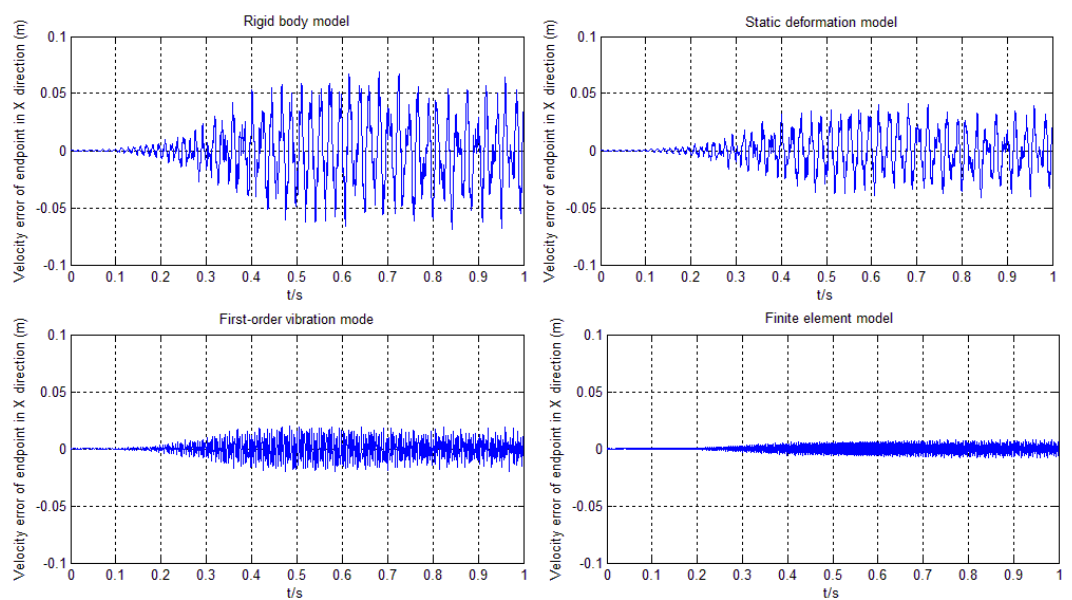

Fig.7 Displacement deviation of endpoint in X
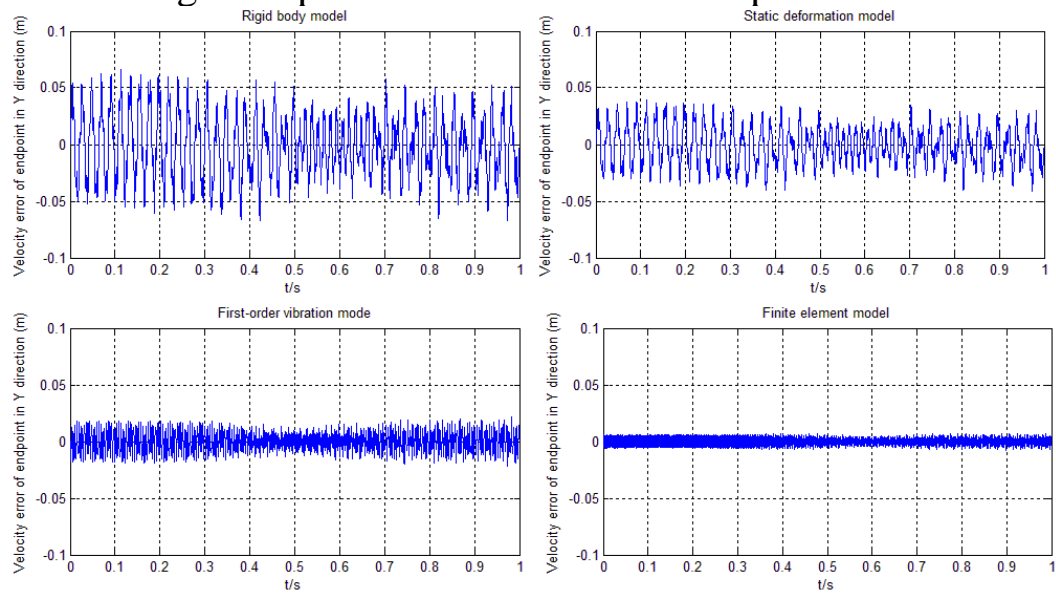

Fig.8 Displacement deviation of endpoint in Y

Through the comparison of the error of terminal displacement in rigid body model and flexible body model under three types of shape function in Figure 7 and Figure 8, it shows that because of the existence of the flexibility of the rod, the terminal velocity of the rod has deviation with the terminal velocity calculated according to the rigidity, and the deviation in $\mathrm{X}$ direction and $\mathrm{Y}$ direction can reach $\pm 0.07 \mathrm{~m} / \mathrm{s}$. In the static deformation model, because the elastic deformation of the rod is considered, because the elastic deformation of the rod is considered, the error decreases compared with the rigid body model; however, the static deformation is taken as the shape function of the flexible body instead of the dynamic deformation, and the calculation error is still large relative to first-order vibration mode and finite element model. The error of first-order vibration mode and finite element model is small, and the computational accuracy increases as the number of mode truncations and the number of divided elements increase; however, the generalized coordinates increase too, and the computational efficiency decreases. In addition, it shows that except that the deformation effect of the connecting rod must be considered, the motion of the rod contains the vibration of the high frequency part, thus to result in the jitter of the rod, and increase the control difficulty of the rod.

\section{Conclusions}

In this paper, aiming at the problem that the shape function affects computational accuracy and efficiency of figures, and based on the analysis of the floating coordinate system established at the specified hinge, the kinetic equation of the flexible multi-body system in general form is first established, three forms of the shape function are discussed respectively and the characteristics of three forms of shape functions are analyzed. The analysis and case show that:

Under the specified floating coordinate system, the decoupling degree of kinetic equation of the flexible body system is the same, namely, mass matrix, stiffness matrix and damping matrix have the same element, showing that the selection of the shape function is not related to the decoupling degree of the equation. The computational accuracy of kinetic equation of the flexible body system has little 
to do with the floating coordinate system and the coupling degree of the equation; the coupling degree mainly affects the complexity of the equation, while the shape function affects computational efficiency and accuracy of the equation greatly.

The form of the equation of the static deformation model is simple, and the computational efficiency is the highest. When calculating the displacement of the rod terminal, the error decreases compared with the rigid body model, and the error at the calculation of the angular velocity is the same as that of the rigid body model. By comparison, the application of finite element model is flexible, which is suitable for the simulation calculation of the complex structure. Different number of mode truncations in the vibration modal model result in different calculation results. The number of generalized coordinates and computational accuracy in finite element model and vibration modal model increase as the number of divided elements and number of mode truncations increase, and the computational efficiency decreases too.

Based on deduction and analysis of the above work from the theoretical formula, and different aspects of the case study, the change rule between the selection of the elastic deformation of the flexible body system and computational accuracy and efficiency of the equation is given in details, which can be used for the reference to simulation and control of the flexible body system dynamics according to different demands.

\section{References}

[1] Deshi Wang:Theory of Gun vibration (Weapons industry press, Beijing 2015,In Chinese)

[2] Popov., E. L.: Dynamics and Its Algorism for Manipulation Robot. (Mechanical industry press , Beijing 1983).

[3] Walker,M.W.,Orin, et al. Efficient dynamic computer simulation of robotic mechanisms, journal of dynamic systems. Measurement and Control, 104(1982), p. 205-211

[4] Deshi Wang. Recursive algorism for the inertia compensation control. Periodic Vibration and Bifurcation in Nonlinear Systems, edtied by Zhang Wei (Science Press, Beijing 2002,In Chinese) .

[5] Kane, T.R., Levinson, et al.in: Submitted to The International Conference on the Applied Mechanics, 9th, Canada(1983)

[6] Witterburg, J.. Dynamics of systems of rigid bodies (B.G. Teubner, Stuttgart 1977).

[7] Robe, T.R., Kane, et al.in: Solid and Structure . 3(1996), p.333-352,691-703,1031-1051.

[8] Huston, R. L., Passerello, et al. Human body dynamics: impact, occupational, and athletic aspects (Claremont press, Oxford 1982).

[9] Deshi Wang in: ASME Design Engineering and Technology Conference/Vib8380, U.S.A. (1999)

[10] Deshi Wang, Yuedong Shi in: Journal of Dynamics and Control, 10(04) (2012), p. 304-323. (In Chinese)

[11] Deshi Wang in: Proceeding of ASME Machine and Mechanism Conference, U.S.A(1993).

[12] Rui Xiaoting in: Acta Weapon Industry. 2(1994), p.1-5. (In Chinese)

[13] Qizhao Zhou in: Advances in Mechanics. 19 (4) (1989), p.464-476,In Chinese.

[14] Qizhao Zhou: An important research direction of multibody system dynamics ( Peking University, Beijing 1988, In Chinese).

[15] Youfang Lu: Flexible Multi-Body System Dynamics (Higher Education Press, Beijing 1993,In Chinese).

[16] Pan Zeng: Finite element analysis and application (Tsinghua university press, Beijing 2003,In Chinese). 